Kragujevac Journal of Mathematics

Volume 45(1) (2021), Pages 115-125.

\title{
HERMITE-HADAMARD TYPE INEQUALITIES FOR OPERATOR GEOMETRICALLY CONVEX FUNCTIONS II
}

\author{
A. TAGHAVI, V. DARVISH, AND T. AZIMI ROUSHAN
}

\begin{abstract}
In this paper, we prove some Hermite-Hadamard type inequalities for operator geometrically convex functions for non-commutative operators.
\end{abstract}

\section{Introduction AND PRELIMINARIES}

Let $B(H)$ stand for $C^{*}$-algebra of all bounded linear operators on a complex Hilbert space $H$ with inner product $\langle\cdot, \cdot\rangle$. An operator $A \in B(H)$ is strictly positive and write $A>0$ if $\langle A x, x\rangle>0$ for all $x \in H$. Let $B(H)^{++}$stand for all strictly positive operators on $B(H)$.

Let $A$ be a self-adjoint operator in $B(H)$. The Gelfand map establishes a *isometrically isomorphism $\Phi$ between the set $C(\operatorname{Sp}(A))$ of all continuous functions defined on the spectrum of $A$, denoted $\operatorname{Sp}(A)$, and the $C^{*}$-algebra $C^{*}(A)$ generated by $A$ and the identity operator $1_{H}$ on $H$ as follows.

For any $f, g \in C(\operatorname{Sp}(A)))$ and any $\alpha, \beta \in \mathbb{C}$ we have:

- $\Phi(\alpha f+\beta g)=\alpha \Phi(f)+\beta \Phi(g)$;

- $\Phi(f g)=\Phi(f) \Phi(g)$ and $\Phi(\bar{f})=\Phi(f)^{*}$;

- $\|\Phi(f)\|=\|f\|:=\sup _{t \in \operatorname{Sp}(A)}|f(t)|$;

- $\Phi\left(f_{0}\right)=1_{H}$ and $\Phi\left(f_{1}\right)=A$, where $f_{0}(t)=1$ and $f_{1}(t)=t$, for $t \in \operatorname{Sp}(A)$.

With this notation we define $f(A)=\Phi(f)$ for all $f \in C(\operatorname{Sp}(A))$, and we call it the continuous functional calculus for a self-adjoint operator $A$. If $A$ is a self-adjoint operator and both $f$ and $g$ are real valued functions on $\operatorname{Sp}(A)$ then the following important property holds: $f(t) \geq g(t)$ for any $t \in \operatorname{Sp}(A)$ implies that $f(A) \geq g(A)$,

Key words and phrases. Operator geometrically convex function, Hermite-Hadamard inequality. 2010 Mathematics Subject Classification. Primary: 47A63. Secondary: 15A60, 47B05.

DOI 10.46793/KgJMat2101.115T

Received: August 19, 2018.

Accepted: October 09, 2018. 
in the operator order of $B(H)$, see [12]. A real valued continuous function $f: \mathbb{R} \rightarrow \mathbb{R}$ is said to be convex (concave) if

$$
f(\lambda a+(1-\lambda) b) \leq(\geq) \lambda f(a)+(1-\lambda) f(b),
$$

for $a, b \in \mathbb{R}$ and $\lambda \in[0,1]$. The following Hermite-Hadamard inequality holds for any convex function $f$ defined on $\mathbb{R}$

$$
\begin{aligned}
(b-a) f\left(\frac{a+b}{2}\right) & \leq \int_{a}^{b} f(x) d x \\
& \leq(b-a) \frac{f(a)+f(b)}{2}, \quad \text { for } a, b \in \mathbb{R} .
\end{aligned}
$$

The author of [8, Remark 1.9.3] gave the following refinement of Hermite-Hadamard inequalities for convex functions

$$
\begin{aligned}
f\left(\frac{a+b}{2}\right) & \leq \frac{1}{2}\left(f\left(\frac{3 a+b}{4}\right)+f\left(\frac{a+3 b}{4}\right)\right) \\
& \leq \frac{1}{b-a} \int_{a}^{b} f(x) d x \\
& \leq \frac{1}{2}\left(f\left(\frac{a+b}{2}\right)+\frac{f(a)+f(b)}{2}\right) \\
& \leq \frac{f(a)+f(b)}{2} .
\end{aligned}
$$

A real valued continuous function is operator convex if

$$
f(\lambda A+(1-\lambda) B) \leq \lambda f(A)+(1-\lambda) f(B),
$$

for self-adjoint operator $A, B \in B(H)$ and $\lambda \in[0,1]$. In [2] Dragomir investigated the operator version of the Hermite-Hadamard inequality for operator convex functions. Let $f: \mathbb{R} \rightarrow \mathbb{R}$ be an operator convex function on the interval $I$ then, for any self-adjoint operators $A$ and $B$ with spectra in $I$, the following inequalities hold

$$
\begin{aligned}
f\left(\frac{A+B}{2}\right) & \leq 2 \int_{\frac{1}{4}}^{\frac{3}{4}} f(t A+(1-t) B) d t \\
& \leq \frac{1}{2}\left[f\left(\frac{3 A+B}{4}\right)+f\left(\frac{A+3 B}{4}\right)\right] \\
& \leq \int_{0}^{1} f((1-t) A+t B) d t \\
& \leq \frac{1}{2}\left[f\left(\frac{A+B}{2}\right)+\frac{f(A)+f(B)}{2}\right] \\
& \leq \frac{f(A)+f(B)}{2} .
\end{aligned}
$$

For the first inequality in above, see [10]. 
A continuous function $f: I \subseteq \mathbb{R}^{+} \rightarrow \mathbb{R}^{+}$( $\mathbb{R}^{+}$denoted positive real numbers) is said to be geometrically convex function (or multiplicatively convex function) if

$$
f\left(a^{\lambda} b^{1-\lambda}\right) \leq f(a)^{\lambda} f(b)^{1-\lambda}
$$

for $a, b \in I$ and $\lambda \in[0,1]$.

The author of $[7, \mathrm{p} .158]$ showed that every polynomial $P(x)$ with non-negative coefficients is a geometrically convex function on $[0, \infty)$. More generally, every real analytic function $f(x)=\sum_{n=0}^{\infty} c_{n} x^{n}$ with non-negative coefficients is geometrically convex function on $(0, R)$ where $R$ denotes the radius of convergence. Also, see $[9,11]$. In [10], the following inequalities were obtained for a geometrically convex function

$$
\begin{aligned}
f(\sqrt{a b}) & \leq \sqrt{\left(f\left(a^{\frac{3}{4}} b^{\frac{1}{4}}\right) f\left(a^{\frac{1}{4}} b^{\frac{3}{4}}\right)\right)} \\
& \leq \exp \left(\frac{1}{\log b-\log a} \int_{a}^{b} \frac{\log f(t)}{t} d t\right) \\
& \leq \sqrt{f(\sqrt{a b})} \cdot \sqrt[4]{f(a)} \cdot \sqrt[4]{f(b)} \\
& \leq \sqrt{f(a) f(b)} .
\end{aligned}
$$

In this paper, we prove some Hermite-Hadamard inequalities for operator geometrically convex functions. Moreover, in the final section, we present some examples and remarks.

\section{Hermite-Hadamard Inequalities for Geometrically Convex FUNCTIONS}

In this section, we introduce the concept of operator geometrically convex function for positive operators and prove the Hermite-Hadamard type inequalities for this function.

Proposition 2.1. Let $A, B \in B(H)^{++}$such that $\operatorname{Sp}(A), \operatorname{Sp}(B) \subseteq I$, and $t \in[0,1]$. Then $\operatorname{Sp}\left(A \sharp_{t} B\right) \subseteq I$, where $A \sharp_{t} B=A^{\frac{1}{2}}\left(A^{-\frac{1}{2}} B A^{-\frac{1}{2}}\right)^{t} A^{\frac{1}{2}}$ is t-geometric mean.

Proof. Let $I=[m, M]$ for some positive real numbers $m, M$ with $m<M$. Since $\operatorname{Sp}(A), \operatorname{Sp}(B) \subseteq I$ it is equivalent to $m 1_{H} \leq A \leq M 1_{H}$ and $m 1_{H} \leq B \leq M 1_{H}$. So, by virtue of the fact that if $a, b$ be self-adjoint operators in $C^{*}$-algebra $\mathcal{A}$ which $a \leq b$ and $c \in \mathcal{A}$, then $c^{*} a c \leq c^{*} b c$, and also by using the operator monotonicity property of the function $f(x)=x^{t}$ on $(0, \infty)$ for $t \in[0,1]$, we get the result.

Now, by applying Proposition 2.1, we present the following definition.

Definition 2.1. A continuous function $f: I \subseteq \mathbb{R}^{+} \rightarrow \mathbb{R}^{+}$is said to be operator geometrically convex if

$$
f\left(A \sharp_{t} B\right) \leq f(A) \sharp_{t} f(B),
$$

for $A, B \in B(H)^{++}$such that $\operatorname{Sp}(A), \operatorname{Sp}(B) \subseteq I$ and $t \in[0,1]$. 
We need the following lemmas for proving our theorems.

Lemma $2.1([4,5])$. Let $A, B \in B(H)^{++}$and let $t, s, u \in \mathbb{R}$. Then

$$
\left(A \sharp_{t} B\right) \sharp_{s}\left(A \sharp_{u} B\right)=A \sharp_{(1-s) t+s u} B \text {. }
$$

Lemma $2.2([4])$. Let $A, B, C$ and $D$ be operators in $B(H)^{++}$and let $t \in \mathbb{R}$. Then, we have

$$
A \sharp_{t} B \leq C \sharp_{t} D
$$

for $A \leq C$ and $B \leq D$.

Lemma 2.3. Let $A, B \in B(H)^{++}$. If $f: I \subseteq \mathbb{R}^{+} \rightarrow \mathbb{R}^{+}$is a continuous function, then

$$
\int_{0}^{1} f\left(A \sharp_{t} B\right) \sharp f\left(A \sharp_{1-t} B\right) d t \leq\left(\int_{0}^{1} f\left(A \sharp_{t} B\right) d t\right) \sharp\left(\int_{0}^{1} f\left(A \sharp_{1-t} B,\right) d t\right)
$$

such that $\operatorname{Sp}(A), \operatorname{Sp}(B) \subseteq I$.

Proof. Since the function $t^{\frac{1}{2}}$ is operator concave, we can write

$$
\left(\left(\int_{0}^{1} f\left(A \sharp_{1-u} B\right) d u\right)^{\frac{-1}{2}}\left(\int_{0}^{1} f\left(A \sharp_{u} B\right) d u\right)\left(\int_{0}^{1} f\left(A \sharp_{1-u} B\right) d u\right)^{\frac{-1}{2}}\right)^{\frac{1}{2}}
$$

(by change of variable $v=1-u$ )

$$
\begin{aligned}
= & \left(\left(\int_{0}^{1} f\left(A \sharp_{v} B\right) d v\right)^{\frac{-1}{2}}\left(\int_{0}^{1} f\left(A \sharp_{u} B\right) d u\right)\left(\int_{0}^{1} f\left(A \sharp_{v} B\right) d v\right)^{\frac{-1}{2}}\right)^{\frac{1}{2}} \\
= & \left(\int_{0}^{1}\left(\int_{0}^{1} f\left(A \sharp_{v} B\right) d v\right)^{\frac{1}{2}} f\left(A \sharp_{u} B\right)\left(\int_{0}^{1} f\left(A \sharp_{v} B\right) d v\right)^{\frac{1}{2}} d u\right)^{\frac{1}{2}} \\
= & \left(\int_{0}^{1}\left(\int_{0}^{1} f\left(A \sharp_{v} B\right) d v\right)^{\frac{-1}{2}}\left(f\left(A \sharp_{1-u} B\right)\right)^{\frac{1}{2}}\left(\left(f\left(A \sharp_{1-u} B\right)\right)^{\frac{-1}{2}} f\left(A \sharp_{u} B\right)\left(f\left(A \sharp_{1-u} B\right)\right)^{\frac{-1}{2}}\right)\right. \\
& \left.\times\left(f\left(A \sharp_{1-u} B\right)\right)^{\frac{1}{2}}\left(\int_{0}^{1} f\left(A \sharp_{v} B\right) d v\right)^{\frac{-1}{2}} d u\right)^{\frac{1}{2}}
\end{aligned}
$$

(by the operator Jensen inequality)

$$
\begin{aligned}
\geq & \int_{0}^{1}\left(\int_{0}^{1} f\left(A \sharp_{v} B\right) d v\right)^{\frac{-1}{2}}\left(f\left(A \sharp_{1-u} B\right)\right)^{\frac{1}{2}}\left(\left(f\left(A \sharp_{1-u} B\right)\right)^{\frac{-1}{2}} f\left(A \sharp_{u} B\right)\left(f\left(A \sharp_{1-u} B\right)\right)^{\frac{-1}{2}}\right)^{\frac{1}{2}} \\
& \times\left(f\left(A \sharp_{1-u} B\right)\right)^{\frac{1}{2}}\left(\int_{0}^{1} f\left(A \sharp_{v} B\right) d v\right)^{\frac{-1}{2}} d u \\
= & \left(\int_{0}^{1} f\left(A \sharp_{v} B\right) d v\right)^{\frac{-1}{2}} \int_{0}^{1}\left(f\left(A \sharp_{1-u} B\right)\right)^{\frac{1}{2}}\left(\left(f\left(A \sharp_{1-u} B\right)\right)^{\frac{-1}{2}} f\left(A \sharp_{u} B\right)\left(f\left(A \sharp_{1-u} B\right)\right)^{\frac{-1}{2}}\right)^{\frac{1}{2}} \\
& \times\left(f\left(A \sharp_{1-u} B\right)\right)^{\frac{1}{2}} d u\left(\int_{0}^{1} f\left(A \sharp_{v} B\right) d v\right)^{\frac{-1}{2}}
\end{aligned}
$$


(by change of variable $u=1-v$ )

$$
\begin{aligned}
= & \left(\int_{0}^{1} f\left(A \sharp_{1-u} B\right) d u\right)^{\frac{-1}{2}} \int_{0}^{1}\left(f\left(A \sharp_{1-u} B\right)\right)^{\frac{1}{2}}\left(\left(f\left(A \sharp_{1-u} B\right)\right)^{\frac{-1}{2}} f\left(A \sharp_{u} B\right)\left(f\left(A \sharp_{1-u} B\right)\right)^{\frac{-1}{2}}\right)^{\frac{1}{2}} \\
& \times\left(f\left(A \sharp_{1-u} B\right)\right)^{\frac{1}{2}} d u\left(\int_{0}^{1} f\left(A \sharp_{1-u} B\right) d u\right)^{\frac{-1}{2}} .
\end{aligned}
$$

So, we obtain

$$
\begin{aligned}
& \left(\left(\int_{0}^{1} f\left(A \sharp_{1-u} B\right) d u\right)^{\frac{-1}{2}}\left(\int_{0}^{1} f\left(A \sharp_{u} B\right) d u\right)\left(\int_{0}^{1} f\left(A \sharp_{1-u} B\right) d u\right)^{\frac{-1}{2}}\right)^{\frac{1}{2}} \\
\geq & \left(\int_{0}^{1} f\left(A \sharp_{1-u} B\right) d u\right)^{\frac{-1}{2}} \int_{0}^{1}\left(f\left(A \sharp_{1-u} B\right)\right)^{\frac{-1}{2}}\left(\left(f\left(A \sharp_{1-u} B\right)\right)^{\frac{-1}{2}} f\left(A \sharp_{u} B\right)\left(f\left(A \sharp_{1-u} B\right)\right)^{\frac{-1}{2}}\right)^{\frac{1}{2}} \\
& \times\left(f\left(A \sharp_{1-u} B\right)\right)^{\frac{1}{2}} d u\left(\int_{0}^{1} f\left(A \sharp_{1-u} B\right) d u\right)^{\frac{-1}{2}} .
\end{aligned}
$$

Multiplying both side of the above inequality by $\left(\int_{0}^{1} f\left(A \sharp_{1-u} B\right) d u\right)^{\frac{1}{2}}$ we obtain

$$
\left(\int_{0}^{1} f\left(A \sharp_{u} B\right) d u\right) \sharp\left(\int_{0}^{1} f\left(A \sharp_{1-u} B\right) d u\right) \geq \int_{0}^{1} f\left(A \sharp_{u} B\right) \sharp f\left(A \sharp_{1-u} B\right) d u .
$$

Before giving our theorems in this section, we mention the following remark.

Remark 2.1. Let $p(x)=x^{t}$ and $q(x)=x^{s}$ on $[1, \infty)$, where $0 \leq t \leq s$. If $f(A) \leq f(B)$ then $\operatorname{Sp}\left(f(A)^{\frac{-1}{2}}(f(B)) f(A)^{\frac{-1}{2}}\right) \subseteq[1, \infty)$. By functional calculus, we have

$$
p\left(f(A)^{\frac{-1}{2}} f(B) f(A)^{\frac{-1}{2}}\right) \leq q\left(f(A)^{\frac{-1}{2}} f(B) f(A)^{\frac{-1}{2}}\right) .
$$

So,

$$
\left(f(A)^{\frac{-1}{2}} f(B) f(A)^{\frac{-1}{2}}\right)^{t} \leq\left(f(A)^{\frac{-1}{2}} f(B) f(A)^{\frac{-1}{2}}\right)^{s} .
$$

Now, we are ready to prove Hermite-Hadamard type inequality for operator geometrically convex functions.

Theorem 2.1. Let $f$ be an operator geometrically convex function. Then, we have

$$
f(A \sharp B) \leq \int_{0}^{1} f\left(A_{\sharp_{t}} B\right) d t \leq \int_{0}^{1} f(A) \sharp_{t} f(B) d t .
$$

Moreover, if $f(A) \leq f(B)$, then we have

$$
\int_{0}^{1} f\left(A \sharp_{t} B\right) d t \leq \int_{0}^{1} f(A) \sharp_{t} f(B) d t \leq \frac{1}{2}((f(A) \sharp f(B))+f(B)),
$$

for $A, B \in B(H)^{++}$. 
Proof. Let $f$ be a geometrically convex function. Then we have

$$
\begin{array}{rlrl}
f(A \sharp B) & =f\left(\left(A \sharp_{t} B\right) \sharp\left(A \sharp_{1-t} B\right)\right) & & \text { (by Lemma 2.1) } \\
& \leq f\left(A \sharp_{t} B\right) \sharp f\left(A \sharp_{1-t} B\right) \quad & (f \text { is operator geometrically convex }) .
\end{array}
$$

Taking integral of the both sides of the above inequalities on $[0,1]$, we obtain

$$
\begin{aligned}
f(A \sharp B) & \leq \int_{0}^{1} f\left(A \sharp_{t} B\right) \sharp f\left(A \sharp_{1-t} B\right) d t \\
& \leq\left(\int_{0}^{1} f\left(A \sharp_{t} B\right) d t\right) \sharp\left(\int_{0}^{1} f\left(A \sharp_{1-t} B\right) d t\right) \quad \text { (by Lemma 2.3) } \\
& =\int_{0}^{1} f\left(A \sharp_{t} B\right) d t \\
& \leq \int_{0}^{1} f(A) \sharp_{t} f(B) d t .
\end{aligned}
$$

For the case $f(A) \leq f(B)$, by applying Remark 2.1 for $s=\frac{1}{2}$, we have

$$
\left(f(A)^{-\frac{1}{2}} f(B) f(A)^{-\frac{1}{2}}\right)^{t} \leq\left(f(A)^{-\frac{1}{2}} f(B) f(A)^{-\frac{1}{2}}\right)^{\frac{1}{2}} .
$$

By integrating the above inequality over $t \in\left[0, \frac{1}{2}\right]$, we obtain

$$
\int_{0}^{\frac{1}{2}}\left(f(A)^{-\frac{1}{2}} f(B) f(A)^{-\frac{1}{2}}\right)^{t} d t \leq \frac{1}{2}\left(f(A)^{-\frac{1}{2}} f(B) f(A)^{-\frac{1}{2}}\right)^{\frac{1}{2}} .
$$

Multiplying both sides of the above inequality by $f(A)^{\frac{1}{2}}$, we have

$$
\begin{aligned}
& \int_{0}^{\frac{1}{2}} f(A)^{\frac{1}{2}}\left(f(A)^{-\frac{1}{2}} f(B) f(A)^{-\frac{1}{2}}\right)^{t} f(A)^{\frac{1}{2}} d t \\
\leq & \frac{1}{2}\left(f(A)^{\frac{1}{2}}\left(f(A)^{-\frac{1}{2}} f(B) f(A)^{-\frac{1}{2}}\right)^{\frac{1}{2}} f(A)^{\frac{1}{2}}\right) .
\end{aligned}
$$

It follows that

$$
\int_{0}^{\frac{1}{2}} f(A) \sharp_{t} f(B) \leq \frac{f(A) \sharp f(B)}{2} .
$$

On the other hand, by considering Remark 2.1 for $s=1$, we have

$$
\left(f(A)^{-\frac{1}{2}} f(B) f(A)^{-\frac{1}{2}}\right)^{t} \leq f(A)^{-\frac{1}{2}} f(B) f(A)^{-\frac{1}{2}} .
$$

Integrating the above inequality over $t \in\left[\frac{1}{2}, 1\right]$, we get

$$
\int_{\frac{1}{2}}^{1}\left(f(A)^{-\frac{1}{2}} f(B) f(A)^{-\frac{1}{2}}\right)^{t} d t \leq \frac{1}{2}\left(f(A)^{-\frac{1}{2}} f(B) f(A)^{-\frac{1}{2}}\right) .
$$

By multiplying both side of the above inequality by $f(A)^{\frac{1}{2}}$, we have

$$
\int_{\frac{1}{2}}^{1} f(A)^{\frac{1}{2}}\left(f(A)^{-\frac{1}{2}} f(B) f(A)^{-\frac{1}{2}}\right)^{t} f(A)^{\frac{1}{2}} d t \leq \frac{f(B)}{2} .
$$


It follows that

$$
\int_{\frac{1}{2}}^{1} f(A) \sharp_{t} f(B) \leq \frac{f(B)}{2} .
$$

From inequalities (2.3) and (2.4) we obtain

$$
\begin{aligned}
\int_{0}^{\frac{1}{2}} f\left(A \sharp_{t} B\right) d t+\int_{\frac{1}{2}}^{1} f\left(A \sharp_{t} B\right) d t & \leq \int_{0}^{\frac{1}{2}} f(A) \sharp_{t} f(B) d t+\int_{\frac{1}{2}}^{1} f(A) \sharp_{t} f(B) d t \\
& \leq \frac{f(A) \sharp f(B)}{2}+\frac{f(B)}{2} .
\end{aligned}
$$

It follows that

$$
\int_{0}^{1} f\left(A \sharp_{t} B\right) d t \leq \int_{0}^{1} f(A) \sharp_{t} f(B) d t \leq \frac{1}{2}((f(A) \sharp f(B))+f(B)) .
$$

By making use of inequalities (2.1) and (2.2), we have the following result.

Corollary 2.1. Let $f$ be an operator geometrically convex function. Then, if $f(A) \leq$ $f(B)$ we have

$$
f(A \sharp B) \leq \int_{0}^{1} f\left(A \sharp_{t} B\right) d t \leq \frac{1}{2}((f(A) \sharp f(B))+f(B)),
$$

for $A, B \in B(H)^{++}$.

Theorem 2.2. Let $f$ be an operator geometrically convex function. Then, we have

$$
f(A \sharp B) \leq \int_{0}^{1} f\left(A \sharp_{t} B\right) \sharp f\left(A \sharp_{1-t} B\right) d t \leq f(A) \sharp f(B),
$$

for $A, B \in B(H)^{++}$.

Proof. We can write

$$
\begin{aligned}
f(A \sharp B) & =f\left(\left(A \sharp_{t} B\right) \sharp\left(A \sharp_{1-t} B\right)\right) \quad \text { (by Lemma 2.1) } \\
& \leq f\left(A \sharp_{t} B\right) \sharp f\left(A \sharp_{1-t} B\right) \quad(f \text { is operator geometrically convex) } \\
& \leq\left(f(A) \sharp_{t} f(B)\right) \sharp\left(f(A) \sharp_{1-t} f(B)\right) \quad \text { (by Lemma 2.2) } \\
& =f(A) \sharp f(B) .
\end{aligned}
$$

So, we obtain

$$
f(A \sharp B) \leq f\left(A \sharp_{t} B\right) \sharp f\left(A \sharp_{1-t} B\right) \leq f(A) \sharp f(B) .
$$

Integrating the above inequality over $t \in[0,1]$ we obtain the desired result.

We divide the interval $[0,1]$ to the interval $[\nu, 1-\nu]$ when $\nu \in\left[0, \frac{1}{2}\right)$ and to the interval $[1-\nu, \nu]$ when $\nu \in\left(\frac{1}{2}, 1\right]$. The we have the following inequalities.

Theorem 2.3. Let $A, B \in B(H)^{++}$such that $f(A) \leq f(B)$. Then, we have

(a) for $\nu \in\left[0, \frac{1}{2}\right)$

$$
f(A) \sharp_{\nu} f(B) \leq \frac{1}{1-2 \nu} \int_{\nu}^{1-\nu} f(A) \sharp_{t} f(B) d t \leq f(A) \sharp_{1-\nu} f(B) ;
$$


(b) for $\nu \in\left(\frac{1}{2}, 1\right]$

$$
f(A) \sharp_{1-\nu} f(B) \leq \frac{1}{2 \nu-1} \int_{1-\nu}^{\nu} f(A) \sharp_{t} f(B) d t \leq f(A) \sharp_{\nu} f(B) .
$$

Proof. Let $\nu \in\left[0, \frac{1}{2}\right)$, then by Remark 2.1 we have

$$
\begin{aligned}
\left(f(A)^{\frac{-1}{2}} f(B) f(A)^{\frac{-1}{2}}\right)^{\nu} & \leq\left(f(A)^{\frac{-1}{2}} f(B) f(A)^{\frac{-1}{2}}\right)^{t} \\
& \leq\left(f(A)^{\frac{-1}{2}} f(B) f(A)^{\frac{-1}{2}}\right)^{1-\nu}
\end{aligned}
$$

for $\nu \leq t \leq 1-\nu$ and $A, B \in B(H)^{++}$such that $\operatorname{Sp}(A), \operatorname{Sp}(B) \subseteq I$.

By integrating the above inequality over $t \in[\nu, 1-\nu]$ we obtain

$$
\begin{aligned}
\int_{\nu}^{1-\nu}\left(f(A)^{\frac{-1}{2}} f(B) f(A)^{\frac{-1}{2}}\right)^{\nu} d t & \leq \int_{\nu}^{1-\nu}\left(f(A)^{\frac{-1}{2}} f(B) f(A)^{\frac{-1}{2}}\right)^{t} d t \\
& \leq \int_{\nu}^{1-\nu}\left(f(A)^{\frac{-1}{2}} f(B) f(A)^{\frac{-1}{2}}\right)^{1-\nu} d t
\end{aligned}
$$

It follows that

$$
\begin{aligned}
\left(f(A)^{\frac{-1}{2}} f(B) f(A)^{\frac{-1}{2}}\right)^{\nu} & \leq \frac{1}{1-2 \nu} \int_{\nu}^{1-\nu}\left(f(A)^{\frac{-1}{2}} f(B) f(A)^{\frac{-1}{2}}\right)^{t} d t \\
& \leq\left(f(A)^{\frac{-1}{2}} f(B) f(A)^{\frac{-1}{2}}\right)^{1-\nu} .
\end{aligned}
$$

Multiplying the both sides of the above inequality by $f(A)^{\frac{1}{2}}$ gives us

$$
f(A) \sharp_{\nu} f(B) \leq \frac{1}{1-2 \nu} \int_{\nu}^{1-\nu} f(A) \sharp_{t} f(B) d t \leq f(A) \sharp_{1-\nu} f(B) .
$$

Also, we know that

$$
\begin{aligned}
\lim _{\nu \rightarrow \frac{1}{2}} f(A) \sharp_{\nu} f(B) & =\lim _{\nu \rightarrow \frac{1}{2}} \frac{1}{1-2 \nu} \int_{\nu}^{1-\nu} f(A) \sharp_{t} f(B) d t \\
& =\lim _{\nu \rightarrow \frac{1}{2}} f(A) \sharp_{1-\nu} f(B) \\
& =f(A) \sharp f(B) .
\end{aligned}
$$

Similarily, for $\nu \in\left(\frac{1}{2}, 1\right]$, by the same proof as above, we get

$$
f(A) \sharp_{1-\nu} f(B) \leq \frac{1}{2 \nu-1} \int_{1-\nu}^{\nu} f(A) \sharp_{t} f(B) d t \leq f(A) \sharp_{\nu} f(B) .
$$

By definition of geometrically convex function and (2.5) we have

$$
\begin{aligned}
f\left(A \sharp_{\nu} B\right) & \leq \frac{1}{1-2 \nu} \int_{\nu}^{1-\nu} f\left(A \sharp_{t} B\right) d t \\
& \leq \frac{1}{1-2 \nu} \int_{\nu}^{1-\nu} f(A) \sharp_{t} f(B) d t \\
& \leq f(A) \sharp_{1-\nu} f(B),
\end{aligned}
$$


for $\nu \in\left[0, \frac{1}{2}\right)$. We should mention here that

$$
\lim _{\nu \rightarrow \frac{1}{2}} \frac{1}{1-2 \nu} \int_{\nu}^{1-\nu} f\left(A \sharp_{t} B\right) d t=\lim _{\nu \rightarrow \frac{1}{2}} f\left(A \sharp_{\nu} B\right)=f(A \sharp B) .
$$

On the other hand, by the definition of geometrically convex function and (2.6) we have

$$
\begin{aligned}
f\left(A \sharp_{1-\nu} B\right) & \leq \frac{1}{2 \nu-1} \int_{1-\nu}^{\nu} f\left(A \sharp_{t} B\right) d t \\
& \leq \frac{1}{2 \nu-1} \int_{1-\nu}^{\nu} f(A) \sharp_{t} f(B) d t \\
& \leq f(A) \sharp_{\nu} f(B),
\end{aligned}
$$

for $\nu \in\left(\frac{1}{2}, 1\right]$.

\section{EXAMPLES AND REMARKS}

In this section we give some examples of the results that obtained in the previous section.

Remark 3.1. For positive $A, B \in B(H)$, Ando proved in [1] that if $\Psi$ is a positive linear map, then we have

$$
\Psi(A \sharp B) \leq \Psi(A) \sharp \Psi(B) .
$$

The above inequality shows that we can find some examples for Definition 2.1 when $f$ is linear.

Example 3.1. It is easy to check that the function $f(t)=t^{-1}$ is operator geometrically convex for operators in $B(H)^{++}$.

Definition 3.1. Let $\phi$ be a map on $C^{*}$-algebra $B(H)$. We say that $\phi$ is 2-positive if the $2 \times 2$ operator matrix $\left[\begin{array}{cc}A & B \\ B^{*} & C\end{array}\right] \geq 0$, then we have $\left[\begin{array}{cc}\phi(A) & \phi(B) \\ \phi\left(B^{*}\right) & \phi(C)\end{array}\right] \geq 0$.

In [6], M. Lin gave an example of a 2-positive map over contraction operators (i.e., $\|A\|<1)$. He proved that

$$
\phi(t)=(1-t)^{-1}
$$

is 2-positive.

Example 3.2. Let $A$ and $B$ be two contraction operators in $B(H)^{++}$. Then it is easy to check $A \sharp B$ is also a contraction and positive. Also, we know the $2 \times 2$ operator matrix

$$
\left[\begin{array}{cc}
A & A \sharp B \\
A \sharp B & B
\end{array}\right]
$$

is semidefinite positive. Hence, by (3.1) we obtain

$$
\left[\begin{array}{cc}
(I-A)^{-1} & (I-(A \sharp B))^{-1} \\
(I-(A \sharp B))^{-1} & (I-B)^{-1}
\end{array}\right]
$$


is semidefinite positive.

On the other hand, by Ando's characterization of the geometric mean if $X$ is a Hermitian matrix and

$$
\left[\begin{array}{ll}
A & X \\
X & B
\end{array}\right] \geq 0
$$

then $X \leq A \sharp B$. So we conclude that $(I-(A \sharp B))^{-1} \leq(I-A)^{-1} \sharp(I-B)^{-1}$. Therefore, the function $\phi(t)=(1-t)^{-1}$ is operator geometrically convex.

Also, Lin proved that the function

$$
\phi(t)=\frac{1+t}{1-t}
$$

is 2-positive over contractions. By the same argument as Example 3.2 we can say the above function is operator geometrically convex too.

Example 3.3. In the proof of [3, Theorem 4.12], by applying Hölder-McCarthy inequality the authors showed the following inequalities

$$
\begin{aligned}
\left\langle A \sharp_{\alpha} B x, x\right\rangle & =\left\langle\left(A^{\frac{-1}{2}} B A^{\frac{-1}{2}}\right)^{\alpha} A^{\frac{1}{2}} x, A^{\frac{1}{2}} x\right\rangle \\
& \leq\left\langle\left(A^{\frac{-1}{2}} B A^{\frac{-1}{2}}\right) A^{\frac{1}{2}} x, A^{\frac{1}{2}} x\right\rangle^{\alpha}\left\langle A^{\frac{1}{2}} x, A^{\frac{1}{2}} x\right\rangle^{1-\alpha} \\
& =\langle A x, x\rangle^{1-\alpha}\langle B x, x\rangle^{\alpha} \\
& =\left\langle A x, x \sharp_{\alpha}\langle B x, x\rangle,\right.
\end{aligned}
$$

for $x \in H$ and $\alpha \in[0,1]$. By taking the supremum over unit vector $x$, we obtain that $f(x)=\|x\|$ is geometrically convex function for usual operator norms.

By the above example and Corollary 2.1, when $\|A\| \leq\|B\|$ we have

$$
\|A \sharp B\| \leq \int_{0}^{1}\left\|A \sharp_{t} B\right\| d t \leq \frac{1}{2}(\sqrt{\|A\|\|B\|}+\|B\|),
$$

for $A, B \in B(H)^{++}$.

\section{REFERENCES}

[1] T. Ando, Concavity of certain maps on positive definite matrices and applications to Hadamard products, Linear Algebra Appl. 26 (1979), 203-241.

[2] S. S. Dragomir, Hermite-Hadamard's type inequalities for operator convex functions, Appl. Math. Comput. 218 (2011), 766-772.

[3] C. Jung, H. Lee, Y. Lim and T. Yamazaki, Weighted geometric mean of n-operators with n-parameters, Linear Algebra Appl. 432 (2010), 1515-1530.

[4] F. Kubo and T. Ando, Means of positive linear operators, Math. Ann. 246(3) (1980), 205-224.

[5] J. Lawson and Y. Lim, Weighted means and Karcher equations of positive operators, Proc. Natl. Acad. Sci. USA 110(39) (2013), 15626-15632.

[6] M. Lin, A Lewent type determinantal inequality, Taiwanese J. Math. 17 (2013), 1303-1309.

[7] C. P. Niculescu, Convexity according to the geometric mean, Math. Inequal. Appl. 3(2) (2000), 155-167. 
[8] C. P. Niculescu and L. E. Persson, Convex Functions and Their Applications: A Contemporary Approach, Springer, Berlin, Heidelberg, New York, Hong Kong, London, Milan, Paris, Tokyo, 2004.

[9] A. Taghavi, V. Darvish, H. M. Nazari and S. S. Dragomir, Hermite-Hadamard type inequalities for operator geometrically convex functions, Monatsh. Math. 181 (2016), 187-203.

[10] V. Darvish, S. S. Dragomir, H. M. Nazari, and A. Taghavi, Some inequalities associated with the Hermite-Hadamard inequalities for operator h-convex functions, Acta Comment. Univ. Tartu. Math. 21(2) (2017), 287-297.

[11] A. Taghavi, T. A. Roushan and V. Darvish, Some refinements for the arithmetic-geometric mean and Cauchy-Schwarz matrix norm interpolating inequalities, Bulletin of the Iranian Mathematical Society 44 (2018), 927-936.

[12] K. Zhu, An Introduction to Operator Algebras, CRC Press, Boca Raton, Florida,1993.

Department of MATHEMATiCs,

FACULTY OF SCIENCE,

University of MazAndaran, P. O. Box 47416-1468, BABolsar, Iran

Email address: taghavi@umz.ac.ir

Email address: vahid.darvish@mail.com

Email address: t.roushan@umz.ac.ir 